\title{
Empleo de Declaraciones Únicas de Aduanas para mejorar el análisis estadístico de las exportaciones peruanas de Harina de Pescado en el período 2007 - 2016
}

\section{Using DBF files with Unique Customs Declarations to enhance statistical analysis of peruvian fishmeal exports in the period 2007-2016}

\author{
Carlos Ricardo Guadalupe Butron ${ }^{1 *}$
}

\begin{abstract}
Resumen
El objetivo de esta investigación fue evaluar la factibilidad y conveniencia de emplear las Declaraciones Únicas de Aduanas (DUAs) para mejorar la información de los análisis estadísticos de las exportaciones peruanas, al incluir la desviación estándar. La metodología consistió en usar la base de datos de las exportaciones que ofrece SUNAT gratuitamente, luego se empleó el Excel (Tablas Dinámicas y Fórmulas) para realizar los estudios correspondientes. Los resultados fueron obtenidos de manera inmediata y sin contratiempo, comprobándose la utilidad y conveniencia de esta metodología. Para la validación de este método se emplearon los datos de exportaciones peruanas totales de harina de pescado para el período 2007 - 2016: se cuantificó el impacto de la variación de precios internacionales en la evolución de las exportaciones en valor; cómo China ha manejado sus precios internacionales, convirtiéndose en el primer mercado de destino y la sostenibilidad de las exportaciones de las principales empresas exportadoras de harina de pescado. Se recomienda la utilización de esta metodología también en los análisis de importaciones peruanas. Palabras clave: Harina de pescado peruana; análisis estadístico; exportaciones peruanas; hojas de cálculo; Declaraciones de Aduanas SUNAT.
\end{abstract}

\begin{abstract}
The objective of this research it was evaluate the feasibility and suitability of using the "Unique Customs Declarations" (DUAS) to improve the information of the statistical analyses of Peruvian exports, by including the standard deviation. The methodology consisted in using the database of exports that SUNAT offers free of charge, then in the Excel (Dynamic Tables and Formulas) to carry out the corresponding studies. The results were obtained immediately and without mishap, checking the usefulness and convenience of this methodology. For the validation of this method, data from Peruvian total exports of fishmeal were used for the period 2007 - 2016: The impact of the international price variation on the evolution of exports in value was quantified; How China has managed its international prices, becoming the first destination market and the sustainability of exports of the main fish meal exporting companies. It is recommended to use this methodology also in the analysis of Peruvian imports.
\end{abstract}

Keywords: Peruvian fish meal; statistical analysis; Peruvian exports; spreadsheets; SUNAT customs declarations.

\section{Introducción}

Los estudios de exportaciones e importaciones usualmente emplean metodologías que usan valores agregados (sumas y promedios), a partir de los cuales extraen las conclusiones y recomendaciones para la toma de decisiones correspondientes (INEI, 2013; BCRP, 2017). Esta situación puede apreciarse en diferentes anuarios estadísticos disponibles (SUNAT, PRODUCE, BCRP). Sin embargo, el principal problema de esta modalidad de análisis es que no se considera la variabilidad que pudiera haber según los diferentes despachos, en diferentes fechas, a diferentes países o por diferentes empresas exportadoras.

Esta investigación se justifica, en primer lugar, porque los datos detallados de los documentos oficiales de exportación (Declaraciones Únicas de Aduanas), están disponibles en el portal internet de la Superintendencia Nacional de Aduanas y Administración Tributaria
(SUNAT) y esta información es de libre disponibilidad. En segundo lugar, porque la evolución de las exportaciones presenta variaciones que no se explican solo con sumas y promedios. Por ejemplo, las exportaciones de Harina de Pescado, cuya producción (más del 95\% del total) se dirige a los mercados internacionales (Produce, 2017) habiéndose exportado en la década 2007 - 2016 un promedio anual de 1,07 millones de TMB (1,366 millones de US\$), según SUNAT (2017). Además, el Ministerio de la Producción del Perú (PRODUCE, 2017) indica que en esta década han sucedido importantes cambios en las principales variables económicas-comerciales que afectaron las exportaciones de este producto: la implantación de un sistema de pesca de cuotas globales a cuotas individuales (2009); el incremento de los precios internacionales, la variación de la demanda en los principales mercados de destino (a partir del año 2010), entre otros. 
El objetivo general de esta investigación fue evaluar la factibilidad de emplear las Declaraciones Únicas de Aduanas (DUAs) para mejorar el análisis estadístico de las exportaciones peruanas de harina de pescado en el período 2007 - 2016. Los objetivos específicos fueron determinar la operatividad para emplear los datos de las DUAs que ofrece SUNAT para determinar totales, promedios y desviación standard de las principales variables que afectan las exportaciones de un producto de exportación; validar la operatividad mediante su uso real en un análisis estadístico típico de cantidades, valores y precios internacionales; la estructura de los mercados de destino y la importancia relativa de las principales empresas exportadoras de harina de pescado y presentar los resultados del análisis estadístico empleado según esta metodología.

\section{Materiales y métodos}

Para los datos correspondientes a las exportaciones peruanas, fueron las estadísticas oficiales de exportación del Perú, provenientes de las Declaraciones Únicas de Aduanas (DUAs) de la Superintendencia Nacional de Aduanas y Administración Tributaria (SUNAT), las que son de libre acceso en el portal institucional de la SUNAT, en su sección "Aduanas - Operatividad Aduanera Descarga de Información - Detallado por partida". Para esta fuente, se consultaron las exportaciones conjuntas de las siguientes subpartidas arancelarias: (1) 2301.20.11.00 que corresponde a harina de pescado con un contenido de grasa superior a $2 \%$ en peso y (2) 2301.20.19.00 que corresponde a harina de pescado con un contenido de grasa inferior o igual a $2 \%$ en peso. Los resultados se brindan en la misma sección, bajo el encabezado "Resultados".

El equipo que se empleó fue una computadora portátil con un procesador Intel Core i5-3317U@170GHz, con una memoria de ocho gigabyte y un sistema operativo Windows 10 de 64 bits. La hoja de cálculo electrónica fue Excel, perteneciente al paquete Office 365, versión 1707.

Para el empleo de la fuente de información, los datos fueron descargados desde el portal de la SUNAT, en archivos electrónicos con un formato denominado DBF (Data Base File), los cuales fueron transformados a un archivo Excel (XLSX). Luego, fueron analizados mediante la función de Tablas Dinámicas (Pivot Tables). También se empleó las funciones estadísticas y numéricas con que cuenta Excel.

El tipo de fuente de datos usado, fue secundaria. Es decir, aquella publicada pero sin fines relacionados al objetivo de la investigación (Lindley, 1985).

La ruta de acceso en el portal de SUNAT para acceder a las estadísticas de "Detallado por partida" fue: http:// www.aduanet.gob.pe/aduanas/informae/aepartmen.htm. También puede llegarse directamente a una ventana ( $\sin$ instrucciones) accediendo a: "http://www.aduanet.gob.pe/ cl-ad-itestdesp/FrmConsultaSumin.jsp?tcon=B". En esta página se llenaron los datos (subpartida arancelaria a 10 dígitos y un año a la vez) y luego se envió el formulario (presionar "Consultar"). Inmediatamente aparece un texto que dice "Requerimiento enviado correctamente con el nombre: 00000000.CON", donde "00000000" es el número de la consulta.

Los resultados se obtienen en: "http://www. aduanet. gob.pe/cl-ad-itsuministro/descargaS01Alias?accion=ca rgarFrmDescargarResultado", que es una página con los resultados de todos los usuarios. Se debe buscar el número de la consulta y se descargará la información solicitada en un archivo DBF, comprimido.

Para la validación del uso de los datos, el diseño de la investigación (de las exportaciones de harina de pescado) fue de tipo cuantitativo, empleándose un Diseño No Experimental Longitudinal, de Tendencia "Estudios que recaban datos en diferentes puntos del tiempo, para realizar inferencias acerca de la evolución, sus causas o sus efectos" (Hernández et al., 2010).

Para la presentación de los resultados, esta se realizó mediante un análisis de exportaciones totales, por países y por empresas (Shumway, 1988).

\section{Resultados y discusión}

Evaluación de la operatividad de uso de los datos de SUNAT

La obtención de datos de SUNAT fue a partir de su página institucional en la sección Aduanas - Operatividad Aduanera - Descarga de Información - Detallado por partida. Se llenó un formulario con los siguientes datos: subpartida arancelaria y fechas. Se envió el formulario y al cabo de un tiempo variable (entre cinco minutos y una hora, aproximadamente) se recibió los resultados de la consulta en un archivo DBF comprimido. El número total de registros para las exportaciones de harina de pescado desde 2006 hasta 2017 fue de 30,474.

La importación de los datos (DBF) a un formato Excel se realizó de manera inmediata y sin contratiempo. El excel abrió el archivo DBF de manera directa. Sin embargo, SUNAT proporciona los datos un año a la vez, por lo que se importó 10 archivos DBF para convertirlos a XLS y luego consolidarlos en un solo archivo. El empleo de las Tablas Dinámicas fue directo: Excel convirtió la base de datos en tablas agregadas, usando la totalidad de los datos. De esta forma se pudo configurar los resultados de las tablas con Promedios y Desviación Standard sin inconvenientes. Adicionalmente, las funciones de promedio y desviación standard, independientes de las Tablas Dinámicas, también funcionaron correctamente y sin inconvenientes.

La modalidad de "valor agregado" empleado comúnmente en los anuarios o boletines estadísticos solo proporcionan las sumas de las exportaciones mensuales y los precios promedios. Cuando se introduce el cálculo de la desviación estándar (esta metodología) se puede apreciar la variabilidad de los resultados y la imperfección de solo trabajar con sumas y promedios. 
Validación de los datos en un análisis típico de exportaciones Los datos de exportaciones de harina de pescado para el período 2007 - 2016 se presentaron en formatos que indican los datos requeridos para las exportaciones totales, por países y por empresas, en una serie de 10 años. Según cada análisis, se presentaron Totales, Promedios y Desviación standard. Debe mencionarse que las variables que proporciona SUNAT son las siguientes: (1) subpartida arancelaria, (2) Fecha de despacho (en año, mes y día), (3) Aduana de salida, (4) País y Puerto de destino, (5) Valor FOB de exportación, (6) Cantidades (Neto, Bruto y Unidades físicas), (7) Exportador (RUC y Nombre comercial) y (8) Descripción específica del producto. Si bien se empleó un análisis típico de exportaciones, esta metodología permite la presentación de múltiples resultados intercambiando y filtrando las variables anteriormente mencionadas.

Resultados del análisis estadístico de exportaciones de harina de pescado para el período 2007 - 2016

Impacto en cantidad, valor y precios internacionales Las exportaciones peruanas de harina de pescado en cantidad, valor y precios de exportación para el período 2007 2016 se muestran en la Tabla 1. Además, se presenta la Desviación Standard de la serie de precios para cada año.

Tabla 1. Exportaciones peruanas de harina de pescado

\begin{tabular}{ccccc}
\hline Años & $\begin{array}{c}\text { Cantidad } \\
(\text { TMB })\end{array}$ & $\begin{array}{c}\text { Valor } \\
\text { (Millones FOB) }\end{array}$ & $\begin{array}{c}\text { Precios } \\
\text { US } / T M)\end{array}$ & $\begin{array}{c}\text { DesvEstp } \\
\text { de Precio }\end{array}$ \\
\hline 2007 & 877,927 & 817 & 930 & 192 \\
2008 & 1564,042 & 1,412 & 912 & 118 \\
2009 & 1541,239 & 1,426 & 939 & 378 \\
2010 & 1086,080 & 1,612 & 1,470 & 355 \\
2011 & 1293,619 & 1,770 & 1,375 & 182 \\
2012 & 1342,880 & 1,781 & 1,328 & 292 \\
2013 & 857,913 & 1,372 & 1,590 & 468 \\
2014 & 855,742 & 1,336 & 1,558 & 290 \\
2015 & 685,695 & 1,135 & 1,577 & 367 \\
2016 & 631,747 & 996 & 1,527 & 484 \\
\hline Total general & 10736,884 & 13,657 & no aplica \\
\hline Promedio & 1073,688 & 1,366 & 1,320 & \\
Desv, Standard & 325,311 & 299 & 267 & \\
\hline Fuente: SUNAT (2017). & \multicolumn{4}{l}{}
\end{tabular}

Se aprecia que la evolución de las cantidades y valores exportados ha sido decreciente, siguiendo la disponibilidad de materia prima, la cual depende de la abundancia de anchoveta y las leyes que regulan su pesca (Ley General de Pesca, 2017; Ley de Cuotas Individuales, 2015). Esto concuerda con los reseñado por PRODUCE (2017).

Sin embargo, la variación de precios internacionales ha afectado la evolución de las exportaciones en valor. Nótese que la evolución de la desviación standard de los precios internacionales dentro del año (columna DesvEstp de Precio) es apenas 192 US\$/TM (15\% del promedio de la serie) para el año 2006 y 118 US\$/TM (9\%) para el año 2010; mientras que para años subsecuentes se llega a variaciones de hasta 468 US\$/TM (35\%) en el año 2013 y 484 US\$/TM (37\%) en el año 2016.

\section{Estructura de mercados de destino}

Las exportaciones peruanas de harina de pescado, expresadas en valor (millones de US FOB) se muestran en la Tabla 2. Puede comprobarse la marcada concentración de los despachos hacia China, en particular a partir del año 2015 en que la participación porcentual supera el 70\%. Los otros países con mayor importancia relativa son Alemania y Japón; los cuales en la década estudiada superaron los US\$ 1,100 millones. Chile, el siguiente destino en importancia, alcanzó US\$ 528 millones. La preferencia de los exportadores peruanos, se explica por la mayor demanda de China, que emplea la harina de pescado como principal proveedor de proteínas para la preparación de alimentos balanceados para animales, en particular, para langostinos.

Tabla2. Exportaciones peruanas de harina de pescado, en Valor (Millones US\$ FOB, por principales Países)

\begin{tabular}{|c|c|c|c|c|c|c|}
\hline \multirow[b]{2}{*}{ Paises } & China & \multirow[t]{2}{*}{ Alemania } & \multicolumn{3}{|c|}{ Japón Chile $\begin{array}{r}\text { Demás } \\
\text { países }\end{array}$} & Total \\
\hline & Valor $\%$ & & Valor & Valor & Valor & Valor \\
\hline 2007 & 32940 & 113 & 123 & 7 & 244 & 817 \\
\hline 2008 & $741 \quad 53$ & 170 & 140 & 29 & 331 & 1,412 \\
\hline 2009 & $\begin{array}{ll}676 \quad 47\end{array}$ & 266 & 112 & 5 & 367 & 1,426 \\
\hline 2010 & $845 \quad 52$ & 183 & 174 & 60 & 350 & 1,612 \\
\hline 2011 & $1,038 \quad 59$ & 169 & 134 & 70 & 359 & 1,770 \\
\hline 2012 & $890 \quad 50$ & 269 & 164 & 73 & 386 & 1,781 \\
\hline 2013 & $866 \quad 63$ & 141 & 81 & 94 & 191 & 1,372 \\
\hline 2014 & 68851 & 180 & 118 & 119 & 231 & 1,336 \\
\hline 2015 & 86676 & 55 & 38 & 41 & 136 & 1,135 \\
\hline 2016 & $711 \quad 71$ & 89 & 36 & 29 & 130 & 996 \\
\hline Total & $7,651 \quad 56$ & 1,636 & 1,119 & 528 & 2,723 & 13,657 \\
\hline
\end{tabular}
Fuente: SUNAT (2017).

La preferencia se explica por los mayores precios que se paga por TM de este producto. Esta situación se aprecia en la Tabla 3, dónde se muestran las exportaciones peruanas de harina de pescado a China, tanto en cantidad (Toneladas), como en valor (Millones de US\$ FOB) y precios (US\$/TM). A fin de apreciar la importancia de los precios como decisor en la preferencia de los exportadores peruanos, se ha agregado con fines comparativos, el precio promedio internacional de las exportaciones totales de Perú al mundo y luego su comparación "Diferencia de precios".

Es notorio que a inicios de la década en estudio, la diferencia de precios era reducida, pero esta ha crecido a partir del año 2010. Es decir, el año inmediato siguiente a la implantación del sistema de cuotas individuales de pesca. A partir de ese año se han mantenido los precios a China superiores al promedio nacional; llegando a un extremo el año 2012.

Las exportaciones peruanas a Alemania, segundo principal país de destino (Tabla 4). En la Tabla 4 se aprecia 
la diferencia de despachos en cantidad y valor para la serie de tiempo analizada; sin embargo es importante precisar lo que sucede con los precios. También, se muestra que los precios promedio anuales que China paga son significativamente superiores, en particular en los años 2015 y 2016, en que se llegaron a diferencias de 303 US\$/TM y 153 US\$/TM, precisamente cuando las concentraciones a China llegaron a 76 y $71 \%$, respectivamente.

Tabla 3. Exportaciones peruanas de Harina de Pescado, a China

\begin{tabular}{|c|c|c|c|c|c|}
\hline Años & $\begin{array}{c}\text { Valor } \\
\text { (Millones } \\
\text { US FOB) }\end{array}$ & $\begin{array}{l}\text { Cantidad } \\
\text { (TMB) }\end{array}$ & $\begin{array}{c}\text { Precio } \\
\text { (US\$/TM) } \\
\text { a China }\end{array}$ & $\begin{array}{c}\text { Precio } \\
\text { (US\$/TM) } \\
\text { al Mundo }\end{array}$ & $\begin{array}{c}\text { Diferencia } \\
\text { precios }\end{array}$ \\
\hline 2007 & 329 & 376,449 & 894 & 930 & $-35,36$ \\
\hline 2008 & 741 & 833,284 & 898 & 912 & $-14,40$ \\
\hline 2009 & 676 & 753,122 & 933 & 939 & $-5,92$ \\
\hline 2010 & 845 & 554,453 & 1,522 & 1,470 & 52,08 \\
\hline 2011 & 1038 & 754,854 & 1,389 & 1,375 & 13,85 \\
\hline 2012 & 890 & 685,177 & 1,318 & 1,328 & $-9,81$ \\
\hline 2013 & 866 & 541,644 & 1,631 & 1,590 & 41,60 \\
\hline 2014 & 688 & 447,603 & 1,574 & 1,558 & 16,33 \\
\hline 2015 & 866 & 510,953 & 1,689 & 1,577 & 112,16 \\
\hline 2016 & 711 & 446,837 & 1,586 & 1,527 & 59,54 \\
\hline $\begin{array}{l}\text { Total } \\
\text { general }\end{array}$ & 7,651 & $5,904,377$ & 1,355 & 1,296 & 58,94 \\
\hline
\end{tabular}

\section{Principales empresas exportadoras}

Las principales empresas peruanas exportadoras de harina de pescado se presentan en la Tabla 5. Se puede apreciar que la empresa TASA (Tecnológica de Alimentos S.A.) es la mayor exportadora, pues concentró en promedio US\$ 334 Millones (25\%) durante el período analizado (2007-2016), sin mayor variación en el período estudiado (desviación standard de 2\%).

Otras empresas importantes son Corporación Pesquera Inca (COPEINCA), con un $11 \%$ de concentración en el periodo analizado. Le siguen Pesquera Diamante (11\%), Pesquera Hayduk (10\%), Austral Group (9\%), Pesquera
Exalmar (8\%) y China Fisheries Group (CFG) Investments (7\%). Las demás empresas representaron un 19\%. En general, estas siete principales empresas representan más del $80 \%$ de las exportaciones totales de harina de pescado en el periodo analizado.

Tabla 4. Exportaciones peruanas de Harina de Pescado, a Alemania

\begin{tabular}{|c|c|c|c|c|c|}
\hline & $\begin{array}{c}\text { Valor } \\
\text { (Millones } \\
\text { US\$ FOB) }\end{array}$ & $\begin{array}{l}\text { Cantidad } \\
\text { (TMB) }\end{array}$ & $\begin{array}{c}\text { Precio } \\
\text { (US\$/TM) }\end{array}$ & $\begin{array}{l}\text { Precio } \\
\text { US\$/TM) }\end{array}$ & $\begin{array}{l}\text { Diferencia } \\
\text { de precios }\end{array}$ \\
\hline Años & & & $\begin{array}{c}\text { A } \\
\text { Alemania }\end{array}$ & $\begin{array}{c}\text { A } \\
\text { China }\end{array}$ & \\
\hline 2007 & 113 & 119,604 & 965 & 894 & 71 \\
\hline 2008 & 170 & 191,901 & 882 & 898 & -16 \\
\hline 2009 & 266 & 270,620 & 991 & 933 & 57 \\
\hline 2010 & 183 & 133,326 & 1,354 & 1,522 & -168 \\
\hline 2011 & 169 & 126,913 & 1,319 & 1,389 & -70 \\
\hline 2012 & 269 & 199,572 & 1,343 & 1,318 & 25 \\
\hline 2013 & 141 & 89,741 & 1,557 & 1,631 & -74 \\
\hline 2014 & 180 & 117,920 & 1,542 & 1,574 & -32 \\
\hline 2015 & 55 & 40,076 & 1,386 & 1,689 & -303 \\
\hline 2016 & 89 & 60,704 & 1,452 & 1,586 & -135 \\
\hline $\begin{array}{l}\text { Total } \\
\text { general }\end{array}$ & 1,636 & $1,350,376$ & 1,223 & 1,355 & -132 \\
\hline
\end{tabular}

\section{Conclusiones}

El empleo de Declaraciones Únicas deAduanas como fuente de información para análisis estadístico de exportaciones es factible $y$, a pesar de la abundancia de registros, no presenta mayores dificultades en el procesamiento de datos, debido al uso de las tablas dinámicas y funciones estadísticas de las hojas de cálculo. Por otra parte, la computadora empleada no requirió de alguna mejora para el procesamiento de datos. El proceso de validación fue exitoso y sus alcances cumplen a cabalidad lo requerido en un análisis estadístico de exportaciones. Los resultados del análisis indican que exportaciones peruanas de harina

Tabla 5. Exportaciones peruanas de Harina de Pescado, por empresa, en Valor (Millones de US\$ FOB)

\begin{tabular}{|c|c|c|c|c|c|c|c|c|c|}
\hline Años & TASA & COPEINCA & DIAMANTE & HAYDUK & AUSTRAL & EXALMAR & CFG INV & DEMAS & Total General \\
\hline 2007 & 195 & 40 & 61 & 62 & 86 & 41 & 59 & 273 & 817 \\
\hline 2008 & 306 & 176 & 131 & 157 & 117 & 83 & 90 & 352 & 1,412 \\
\hline 2009 & 322 & 177 & 163 & 125 & 160 & 110 & 89 & 282 & 1,426 \\
\hline 2010 & 396 & 205 & 185 & 109 & 139 & 159 & 114 & 305 & 1,612 \\
\hline 2011 & 441 & 202 & 204 & 151 & 169 & 150 & 141 & 312 & 1,770 \\
\hline 2012 & 427 & 241 & 197 & 147 & 160 & 162 & 134 & 314 & 1,781 \\
\hline 2013 & 325 & 175 & 160 & 138 & 134 & 117 & 109 & 215 & 1,372 \\
\hline 2014 & 342 & 185 & 157 & 109 & 107 & 131 & 113 & 193 & 1,336 \\
\hline 2015 & 321 & 137 & 151 & 99 & 96 & 103 & 96 & 132 & 1,135 \\
\hline 2016 & 260 & 58 & 115 & 249 & 83 & 92 & 4 & 134 & 996 \\
\hline Total general & 3,336 & 1,595 & 1,524 & 1,345 & 1,250 & 1,148 & 948 & 2,511 & 13,657 \\
\hline Promedio & 334 & 159 & 152 & 135 & 125 & 115 & 95 & 251 & 1,366 \\
\hline Desv Standard & 70 & 61 & 40 & 47 & 30 & 36 & 38 & 74 & 299 \\
\hline
\end{tabular}

Fuente: SUNAT (2017). 
de pescado, tanto en valor como en cantidad, mostraron un comportamiento decreciente en el periodo estudiado. En cambio, la evolución de los precios de exportación ha sido creciente. China es el principal comprador de la harina de pescado peruana, concentrando un $71 \%$ de las exportaciones peruanas en 2016, mientras que los más cercanos competidores (Alemania y Japón) representaron un $20 \%$ de este total. El principal argumento de China es su capacidad de pagar precios internacionales superiores a sus competidores en hasta US 112/TM (año 2015). Las siete primeras empresas exportadoras concentraron un $80 \%$ del total exportado en el periodo analizado, siendo la empresa TASA la principal exportadora, con un $24.4 \%$ de ese total. Las exportaciones de este grupo han sido consistentes mostrando una baja desviación standard (2\% a $4 \%$ ).

\section{Literatura citada}

Banco Central de Reserva del Perú. 2017. Disponible En: http://www.bcrp.gob.pe/

Hernández, R.; Fernández, C. \& Baptista, P. 2010. Metodología de la investigación. Lima, Perú: McGraw- Hill.

INEI. 2013. Metodología de Cálculo Mensual de los Índices de Precios de Comercio Exterior. Lima, Perú. Autores.

Ley de Cuotas Individuales. 2015. Disponible en: https://www.ey.com/pe/es/newsroom/newsroom-amleycuotas-empresas-pesqueras

Ley General de Pesca. 2017. Decreto Supremo No 0122001-PE. Disponible en: https://www.produce.gob.pe/ landing/pescayconsumoresponsable/ley-general-depesca.pdf

Lindey, D.V. 1985. Reconciliation Of Probability Distributions Research, 31, 866-880.

PRODUCE. 2017. Boletín estadístico pesquero. Lima, Perú: Ministerio de la Producción.

Shumway, R. H. 1988. Applied statistical time series analysis. Englewood Cliffs, NJ: Prentice Hall.

SUNAT. 2017. Operatividad Aduanera [Software de computación]. Lima, Perú: SUNAT.

Centro de Investigación de la Universidad del Pacífico. 2012. La continuidad y el desempeño exportador de la empresa peruana. En Documentos de Discusión DD/12/15 Lima, Perú. 